\title{
Cost-effective and time-saving three-dimensional printing protocol of intra-abdominal cavity of liver transplantation recipient to minimize risk of large-for-size syndrome: the initial experience
}

\author{
Sunghae Park, Mi Seung Kim, Gyu-Seong Choi, Jong Man Kim, Sanghoon Lee, Jae-Won Joh, Jinsoo Rhu
}

Department of Surgery-Transplantation, Samsung Medical Center, Seoul, Korea

Background: The application of three-dimensional (3D) printing has been increasing and we invented a protocol for a cost-effective and time-saving 3D model of intra-abdominal cavity to prevent large-for-size syndrome during liver transplantation.

Methods: Three-dimensional printing of the intra-abdominal cavity were performed on potential adult recipients with small cavity and pediatric patients scheduled for transplantation during the period of July 2020 to July 2021. To reduce time and cost, boundaries of intra-abdominal cavity were outlined based on computed tomography of the potential transplantation recipient with a 1 to $3 \mathrm{~cm}$ distances. The printed pieces were reassembled with a pillar and footing. The printed models of adult patients were used for comparing the size to the graft during deceased donor operation while models of pediatric patients were used for directly comparing the size to the 3D printed graft of living donors.

Results: During the study period, seven adults and five pediatric patients were included. Median time for model production was 10.8 hours (interquartile range [IQR], 9.5-11.8) and estimated median cost for the filament used was 1.64 dollars (IQR, 1.5-1.74). Transplantation of reduction graft $(n=1)$, whole liver transplantation after giving up the previous donor match $(n=2)$, whole liver transplantation from the first matched donor $(n=3)$, and waiting for another donor match after giving up first matched donor $(n=1)$ were resulted using 3D printed model in adult patients. Among pediatric patients, two cases were resulted in reduction graft as planned during preoperative planning and three cases resulted in extended left lateral graft transplantation. All the cases ended up with successful closure of the abdomen with no large-for-size syndrome.

Conclusions: Our cost-effective and time-saving 3D printed model of intra-abdominal cavity was feasible and proved to be useful for preventing large-for-size syndrome in small adult recipients and pediatric patients.

Corresponding author: Jinsoo Rhu

E-mail: jsrrules@gmail.com

(c) The Korean Society for Transplantation

This is an Open Access article distributed under the terms of the Creative Commons Attribution Non-Commercial License (http://creativecommons.org/licenses/by-nc/4.0/) which permits unrestricted non-commercial use, distribution, and reproduction in any medium, provided the original work is properly cited. 